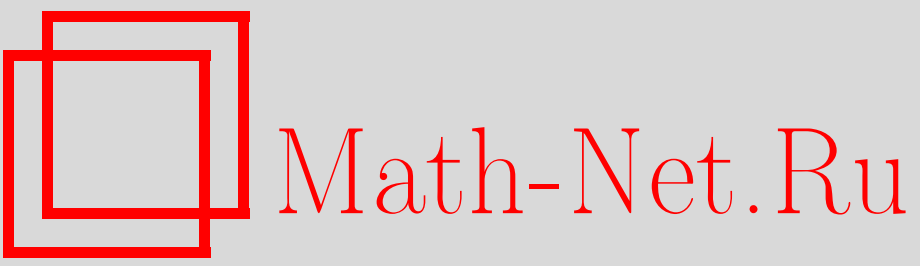

Д. И. Пионтковский, О дифференциально градуированных алгебрах Ли, $У М H$, 2003, том 58, выпуск 1, 193-194

DOI: https://doi.org/10.4213/rm604

Использование Общероссийского математического портала Math-Net.Ru подразумевает, что вы прочитали и согласны с пользовательским соглашением

http://www.mathnet.ru/rus/agreement

Параметры загрузки:

IP: 54.80 .73 .141

26 апреля 2023 г., 18:23:22 


\title{
О ДИФФЕРЕНЦИАЛЬНО ГРАДУИРОВАННЫХ АЛГЕБРАХ ЛИ
}

\author{
Д. И. Пионтковский
}

Предположим, что к градуированной или дифференциально градуированной (д.г.) алгебре Ли $^{1}$ присоединяется несколько свободных переменных и диффференцирование продолжается на всю получившуюся алгебру. В этой заметке изучается вопрос о том, как связаны алгебры гомологий этих двух д.г. алгебр. Такая ситуация возникает, например, при построении минимальной модели Квиллена в рациональной теории гомотопий. Здесь рассматривается (главным образом) случай, когда исходная алгебра - обычная градуированная, т.е. д.г. с нулевым дифференциалом.

Аналогичная "ассоциативная" конструкция, когда к ассоциативной (дифференциально) градуированной алгебре присоединяется свободное множество переменных, изучалась И. Р. Шафаревичем, Е. С. Голодом и другими [1]-[5] и носит название комплекса Шафаревича. Здесь результаты о комплексах Шафаревича ассоциативных д.г. алгебр (полученные, в основном, Е. С. Голодом) переносятся на случай д.г. алгебр Ли. Ссылка в фигурных скобках после каждого утверждения указывает на источник его "ассоциативной" версии. Доказательства, за исключением несколшких тонких моментов, основьваются на применении функтора универсалннй обертьвающей или на почти буквальном воспроизведении "ассоциативных" рассуждений и здесь не приводятся.

Заффиксируем основное поле $k$ нулевой характеристики. Если $x$ - однородньй элемент д.г. векторного пространства (алгебры, модуля), то через $\widetilde{x}$ обозначим свободную переменную той же основной степени и на единицу большей гомологической степени; для множества $X=\left\{x_{i}\right\}$ таких элементов положим $\widetilde{X}=\left\{\widetilde{x}_{i}\right\}$. Через $\mathbb{L}(X)$ (соответственно $\left.T(\widetilde{X})\right)$ обозначается свободная лиева (ассоциативная) супералгебра, порожденная $\widetilde{X}$, а для лиевой или ассоциативной алгебры $A$ через $A\langle\widetilde{X}\rangle$ обозначается свободное (прямое) произведение $A * \mathbb{L}(\widetilde{X})($ соответственно $A * T(\widetilde{X}))$. Комплексом Шафаревича лиевой или ассоциативной д.г. алгебры $A$ по однородному подмножеству ее циклов $X \subset A$ назьвается алгебра $A\langle\widetilde{X}\rangle$ с дифференцированием, продолжающим диффференцирование алгебры $A$ по правилу $d \widetilde{x}_{i}=x_{i}$. Такой комплекс обозначается как $\operatorname{Sh}(X, A)$, а его алгебра гомологий - как $H(X, A)$. Это определение является частным случаем конструкции комплекса Шаффаревича для произвольного многообразия алгебр [6]. В частности, для любой д.г. алгебры Ли $L$ справедливы изоморфиизмы $U \operatorname{Sh}(X, L)=\operatorname{Sh}(X, U L)$ и $U H(X, L)=H(X, U L)$.

Пусть $A$ - градуированная ассоциативная или лиева (супер)алгебра, $f \in A$ - подмножество однородных элементов. $f$ называется сильно свободным или инертным, если комплекс $\operatorname{Sh}(f, A)$ ацикличен в положительных степенях. Если $I$ - идеал, порожденный $f, \operatorname{gr} A$ - ассоциированная градуированная алгебра к $I$-адической фильтрации на $A$, то инертные множества характеризуются также тем, что естественная сюръекция $\chi:(A / I)\langle f\rangle \rightarrow \operatorname{gr} A$ является изоморфизмом [2], [7].

ПреДЛОЖЕНИЕ $1\{[3]\}$. Пусть $f: A \rightarrow B-$ морфизм д.г. алгебр Ли, $x \subset Z B . E c л u f$ индуцирует изморофизм $H A \rightarrow H(x, B)$, то отображение $\rho: H A\langle x\rangle \rightarrow H B$, продолжающее $f_{*}: H A \rightarrow H B,-$ тоже изоморфизм.

Пусть идеал $J$ в градуированной алгебре Ли $A$ минимально порожден множеством $y=\left\{y_{\alpha}\right\}$, $B=A / J$, причем $y$ лежит в идеале $I$, порожденном множеством $x$ элементов положительной степени, $\bar{x}$ - образ множества $x$ в $B$. Тогда существует отображение $\rho: H(x, A)\langle\widetilde{y}\rangle \rightarrow H(\bar{x}, B)$, инду-

Работа выполнена при частичной поддержке Российского фонда фундаментальных исследований (грант № 02-01-00468).

${ }^{1}$ Дифференциально градуированной здесь назьвается биградуированная дифференциалная алгебра с двумя градуировками (возможно, вырожденными): гомологической $\mathbb{Z}_{+}$-градуировкой, которая при действии дифференциала понижается на единицу и задает четность элементов, и основной односвязной $\mathbb{Z}_{+}$-градуировкой такой, что все биградуированные компоненты имеют конечную размерность. 
цированное на $H(x, A)$ естественным отображением $f: A \rightarrow B$ и отправляющее каждьй элемент $\widetilde{y}_{\alpha}$ в элемент $(\operatorname{Sh} f)\left(z_{\alpha}\right)$, где $z_{\alpha} \in \operatorname{Sh}_{1}(x, A)$ - какой-нибудь прообраз элемента $y_{\alpha}$ при дифференцировании. Кроме того, можно рассмотреть отображение $\tau: \operatorname{Sh}(x, A) \rightarrow \operatorname{Sh}(z, \operatorname{Sh}(\bar{x}, B))-$ композицию морфизма $\operatorname{Sh} f$ и вложения $\operatorname{Sh}(\bar{x}, B) \rightarrow \operatorname{Sh}(z, \operatorname{Sh}(\bar{x}, B))$.

Теорема $2\{[3]\}$. Следующие условия әквивалентны:

1) множество у инертно в $A$;

2) отображение $\tau$ индуцирует изоморфизм в гомологиях;

3) отображение $\rho$ биективно;

4) отображение $\rho$ инбективно на әлементах гомологической степени 1 и сюрвективно на әлементах гомологической степени 2.

Назовем полным пересечнием факторалгебру свободной алгебры Ли по идеалу, порожденному инертным множеством (т.е. градуированную алгебру Ли, универсальная обертывающая которой имеет глобальную размерность два).

СлеДСтвИЕ $3\{[3]\}$. Пусть $L-$ градуированная алгебра Ли, порожденная множеством $x$ әлементов положительной степени. Тогда следующие условия әквивалентны:

1) $L$ - полное пересечение;

2) $H(x, L) \simeq \mathbf{L}\left(H_{1}(x, L)\right)$;

3) $H(z, \operatorname{Sh}(x, L))=0$, где $z$-множество ичиллов в $\operatorname{Sh}_{1}(x, L)$, чей образ составляет базис пространства $H_{1}(x, L)$;

4) $H_{2}(x, L)=\left[H_{1}(x, L), H_{1}(x, L)\right]$.

Пусть теперь $L$ - факторалгебра свободной алгебры Ли $A=\mathbf{L}\langle x\rangle$ по однородному идеалу $I$, порожденному множеством $f$. Пусть $\phi$ - минимальное порождающее множество ядра сюръекции $\chi: L\langle f\rangle \rightarrow \operatorname{gr} A$ (как идеала в $L\langle f\rangle)$. Если $f$ инертно, то комплекс $\operatorname{Sh}(f, A)$ ацикличен в положительных степенях, т.е. $H(f, A)=L$, и $\phi$ пусто. В общем случае имеет место

Tеорема 4. а) Алгебра $H(f, A)$ порождается алгеброй $L=H_{0}(f, A)$ и L-подмодулем $H_{1}(f, A)\{[4]\}$.

b) $L$-модуль $H_{1}(f, A)$ минимальным образом порождается образом $\bar{\phi}$ множества $\phi$ в $H(f, A)$, причем векторное пространство порождающих $k \bar{\phi}$ изоморфно $\operatorname{Tor}_{3}^{U L}(k, k)$ $\{[5]\}$.

Для инертного множества $f$ алгебра $H(f, A)$ минимальная из возможных. Ниже описьвается "максимальньй" случай.

СледСТВиЕ $5\{[5]\}$. Предположим, что множество $f$ не является инертным.

Тогда следующие условия эквивалентнь:

1) $H(f, A) \simeq L\langle\phi\rangle$;

2) глобальная размерность универсальной обертывающей $U(L)$ равна 3 ;

3) множество $\phi \subset L\langle f\rangle$ инертно.

\section{СПИСОК ЛИТЕРАТУРЫ}

[1] Е.С. Голод, И.Р. Шафаревич // Изв. АН СССР. Сер. матем. 1964. Т. 28. № 2. C. 261-272. [2] D. Anick // J. Algebra. 1982. V. 78. P. 120-140. [3] Е. С. Голод // Фундам. прикл. матем. 1999. Т. 5. № 1. С. 85-95. [4] Е. С. Голод // Фундам. прикл. матем. 1999. Т. 5. № 1. С. 97-100. [5] Д. И. Пионтковский // Изв. РАН. Сер. матем. 2001. Т. 65. № 3. С. 139-152. [6] Д. И. Пионтковский // Матем. сб. 1998. Т. 189. №11. С. 103-120. [7] S. Halperin, J.-M. Lemaire // Math. Scand. 1987. V. 61. № 1. P. 39-67. 